\title{
Syntheses and Properties of Related Polyoxadiazoles and Polytriazoles
}

\author{
E. R. HENSEMA, ${ }^{\star}$ M. E. R. SENA, ${ }^{2}$ M. H. V. MULDER, ${ }^{1 \dagger}$ and C. A. SMOLDERS ${ }^{1}$ \\ 'University of Twente, Department of Chemical Technology, P.O. Box 217, 7500 AE Enschede, The Netherlands \\ ${ }^{2}$ Instituto de Macromolécules, Universidade do Rio de Janeiro/UFRJ, Ilha do Funao, Centro de Tecnologia, CEP \\ 21945, Rio de Janeiro, Brasil
}

\begin{abstract}
SYNOPSIS
New aromatic poly-1,2,4-triazoles and poly-1,3,4-oxadiazoles are studied as thermally stable membrane materials. Various groups were introduced onto the pendant phenyl groups of poly-1,2,4-triazoles. Glass transition temperature, degradation temperature, and cold crystallization behavior were studied as a function of these groups. Cold crystallization appeared to be highly sensitive to macromolecular regularity. The solubility of poly-1,3,4-oxadiazoles was highly improved upon incorporation of 5-t-butylisophthalic, 1,1,3-trimethyl-3-phenylindane, 4,4'-(2,2'-diphenyl) hexafluoro propane, and diphenyl ether groups into the polymeric main chain, whereas the high glass transition temperatures and degradation temperatures typical for aromatic poly-1,3,4-oxadiazoles were maintained. (c) 1994 John Wiley \& Sons, Inc.
\end{abstract}

Keywords: poly-1,3,4-oxadiazoles $\bullet$ polyhydrazide $\bullet$ poly-1,2,4-triazoles $\bullet$ cold crystallization

\section{INTRODUCTION}

Aromatic poly-1,2,4-triazoles and poly-1,3,4-oxadiazoles are studied as new thermally stable membrane materials for gas separation applications, especially poly [ $p$-, $m$-phenylene- (4-phenyl)-1,2,4-triazole ], which is known for its excellent gas separation properties. ${ }^{1}$ The use of these polymers as membrane materials is hampered due to their poor solubility, which limits the number of possibilities of fabricating membranes with a desired morphology, like asymmetric structures. For example, poly $(p-, m-$ phenylene ) -1,3,4-oxadiazole is soluble only in concentrated sulphuric acid, which is a very difficult solvent to handle in practice.

In this article we wish to report the syntheses and physical properties of poly-1,3,4-oxadiazoles and poly-1,2,4-triazoles where various functional groups are incorporated into and onto the polymer backbone with the aim of increasing their solubility while

\footnotetext{
* Present address: CIBA-GEIGY AG, Central Research Laboratories, $\mathrm{CH}-4002$ Basel, Switzerland.

† To whom correspondence should be addressed.

Journal of Polymer Science: Part A: Polymer Chemistry, Vol. 32, 527-537 (1994) (C) 1994 John Wiley \& Sons, Inc. CCC 0887-624X/94/030527-11
}

maintaining their thermal stability. The relationship between the gas separation properties and these molecular structures was also studied and is reported elsewhere. $^{2}$

The solubility of polymers is often increased when flexible bonds, large pendant groups, or polar substituents are incorporated in the polymer backbone. The introduction of large pendant bulky groups along the polymer backbone results in a less ordered polymer matrix increasing the solubility characteristics. This is nicely illustrated by the fact that poly ( $p$-, $m$-phenylene ) -1,3,4-oxadiazoles are soluble only in concentrated sulphuric acid while poly $[p-$, $m$-phenylene- (4-phenyl)-1,2,4-triazoles ], having an extra pendant phenyl group, are also soluble in $m$ cresol and formic acid.

Solubility is also highly increased if, instead of aromatic groups, aliphatic groups are incorporated into the polymer backbone, but a significant reduction in thermal stability is also observed. Incorporation of aliphatic groups for that reason was avoided. (Nonetheless, cyclohexane was incorporated as a comparison to the aromatic poly $\left(p^{-}, m\right.$ phenylene-1,3,4-oxadiazoles.)

A number of authors have reported poly (1,3,4oxadiazoles) with increased solubility characteris- 
tics. Iwakura has, for example, reported poly-1,3,4oxadiazoles where the phenylene ring has been replaced by a pyridine ring. These polymers are soluble in formic acid, DMF and DMSO. However, these polymers were not synthesized and studied here, since low inherent viscosities were reported. ${ }^{3}$

Russian workers have developed so called "Cardo" poly-1,3,4-oxadiazoles containing a bulky phthalide group. Poly-1,3,4-oxadiazoles containing this group have been studied extensively and the preparation of porous membranes of poly-1,3,4-oxadiazoles containing this group has been patented. ${ }^{4}$

The functional groups that were incorporated into poly-1,3,4-oxadiazoles and poly-1,2,4-triazoles in this study, together with their syntheses, are represented in Figure 1.

A number of reaction routes are known for the preparation of these polymers. ${ }^{5}$ Poly $[p-, m$-phenylene-(4-phenyl) -1,2,4-triazoles] with alternating para- and meta-phenylene groups were prepared by means of a reaction of aniline and aniline derivatives with poly ( $p-, m$-phenylene) hydrazide in polyphosphoric acid [ see Fig. 1 (A)]. Holsten and Lilyquist have studied this reaction extensively using polyhydrazide as a precursor polymer in combination with aniline. ${ }^{6}$ Poly ( $p$-, $m$-phenylene) hydrazide is prepared by means of a polycondensation reaction between terephthaloyl chloride and isophthaloyl dihydrazide.

High molecular weight aromatic poly-1,3,4-oxadiazoles were prepared directly using various dicarboxylic acids and hydrazine sulphate as monomers [see Fig. 1 (B)]. This reaction route was originally reported by Iwakura et $\mathrm{al}^{8}$

\section{EXPERIMENTAL}

\section{Materials}

A mixture of cis- and trans-1,4-cyclohexane dicarboxylic acid was purchased from Aldrich. The pure cis- $(c-\mathrm{CH})$ and the trans-isomer $(t-\mathrm{CH})$ were

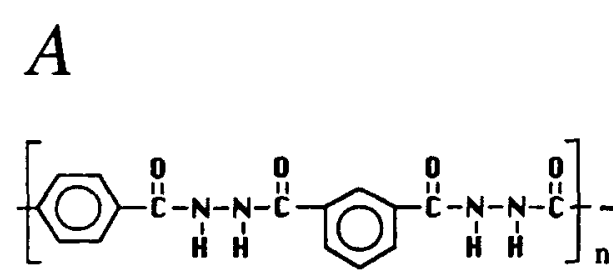

Poly(p-, m-phenylene $)$ hydrazide

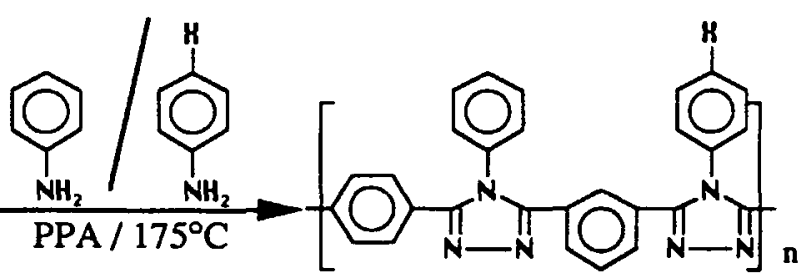

Polylp-, m-phenylene-(4-phenyl)-1,2,4-triazole]

$\mathrm{K}=\mathrm{H}, \mathrm{F}, \mathrm{Cl}$ or $\mathrm{CH}_{3}$

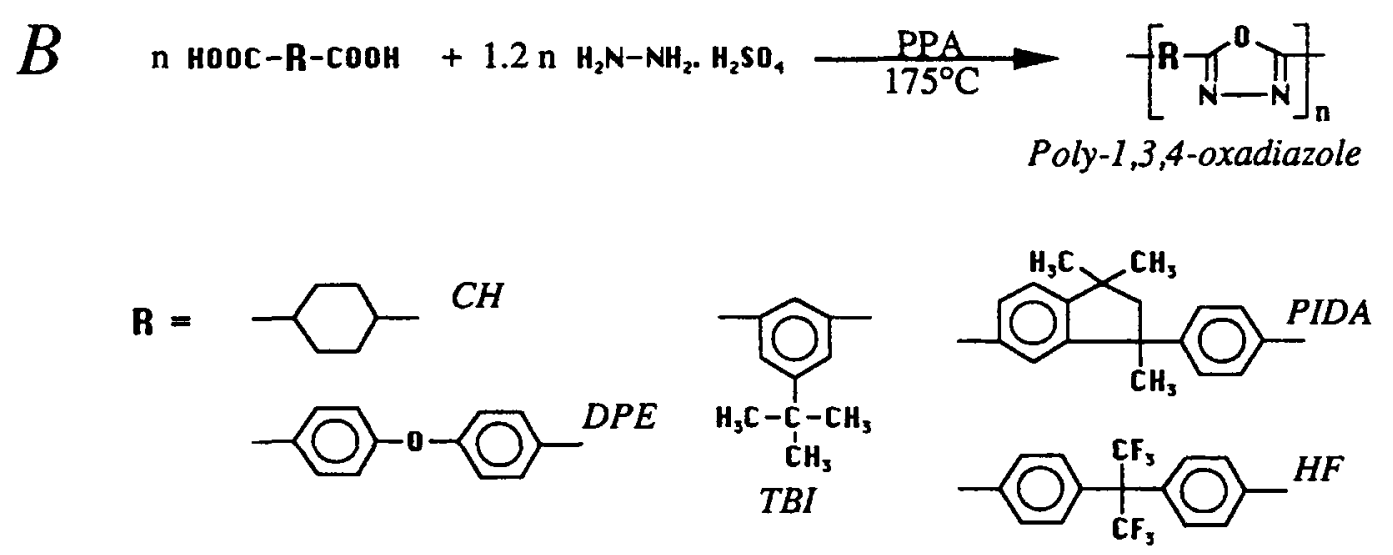

Figure 1. (A) Preparation of aromatic poly [ $p$-, $m$-phenylene(4-phenyl)-1,2,4-triazoles] out of a polyhydrazide in a reaction medium consisting of polyphosphoric acid and mixtures of aniline and para-substituted aniline derivatives. (B) Preparation of poly-1,3,4-oxadiazoles using dicarboxylic acids, $\mathrm{R}(\mathrm{COOH})_{2}$, and hydrazine sulphate as monomers. 
obtained from this mixture by means of a treatment with chloroform. ${ }^{9}$

The dicarboxylic acids, 4,4'-diphenyl ether (DPE) , 1,1,3-trimethyl-3-phenylindan-4',5-dicarboxylic acid (PIDA), and 5-t-butylisophthalic acid (TBI) are Amoco products and 4,4'-(2,2'-diphenyl) hexafluoropropane dicarboxylic acid ( $\mathrm{HF}$ ) was supplied by Hoechst. These dicarboxylic acids were used as received.

All other chemicals were supplied by Merck and were used as received, except NMP, which was distilled under reduced pressure over $\mathrm{CaH}_{2}$, lithium chloride, and dried in a vacuum oven at $150^{\circ} \mathrm{C}$, and aniline, which was distilled under reduced pressure and its colorless distillate stored under nitrogen in a sealed flask in a refrigerator.

Isophthaloyl dihydrazide was prepared from isophthaloyl dimethyl ester and hydrated hydrazine. ${ }^{10}$

\section{Poly ( $p-, m$-phenylene) hydrazide Synthesis 7,10}

Isophthaloyl dihydrazide, $74.24 \mathrm{~g}(0.382 \mathrm{~mol})$ and $16.21 \mathrm{~g} \mathrm{LiCl}$ are dissolved in a 3-necked flask containing $600 \mathrm{~mL}$ of $\mathrm{NMP}$ at $60^{\circ} \mathrm{C}$ under dry nitrogen. After dissolution, the stirred solution is cooled to

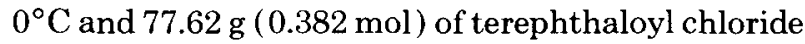
is added in four equal portions. Each following portion is added when the terephthaloyl chloride of the previous portion has completely dissolved. Total addition lasts approximately $2 \mathrm{~h}$. The viscous solution is stirred overnight, and the polymer is precipitated and washed in hyperfiltrated water. After additional washing with ethanol, the polymer is dried in a vacuum oven at $150^{\circ} \mathrm{C}$ for at least $24 \mathrm{~h}$.

Two batches of polyhydrazide were prepared having inherent viscosities of 0.86 and $0.82 \mathrm{dL} / \mathrm{g}$, respectively (0.5 g polymer solution in $100 \mathrm{~mL}$ DMSO).

\section{Poly-1,2,4-triazole Synthesis Using Polyhydrazide as a Precursor Polymer ${ }^{6}$}

In a 3-necked flask, $310 \mathrm{~g}$ polyphosphoric acid (PPA) is heated to $150^{\circ} \mathrm{C}$ under nitrogen. Aniline, $76.65 \mathrm{~g}(0.823 \mathrm{~mol})$, is added dropwise to the stirred solution, while maintaining the temperature at $150^{\circ} \mathrm{C}$. After addition, the temperature is raised to $175^{\circ} \mathrm{C}$, and $10 \mathrm{~g}$ poly $(p-, m$-phenylene $)$ hydrazide is added. The reaction mixture is kept at this temperature for $140 \mathrm{~h}$ and poured into hyperfiltrated water and neutralized with sodium hydroxide under stirring. The product is washed in a $5 \%$ sodium hydroxide solution and washed twice in hyperfiltrated water. Subsequently, the product is extracted with ethanol using a Soxhlet extractor after which it is dried in a vacuum oven at $150^{\circ} \mathrm{C}$ for $24 \mathrm{~h}$. The product is dissolved in formic acid and insoluble parts are removed by means of filtration over a $5 \mu \mathrm{m}$ filter. These solutions are directly used for poly-1,2,4-triazole film preparation, as will be discussed below.

The polymers were obtained in a good yield. Instead of aniline, also mixtures of aniline and aniline derivatives have been employed. In all cases, a ratio of $0.823 \mathrm{~mol}$ aniline and aniline derivative per 310 g PPA has been applied.

\section{Poly-1,3,4-oxadiazole Synthesis ${ }^{8}$}

In a 3-necked flask under a dry nitrogen flux, 0.05 mol dicarboxylic acid and $0.06 \mathrm{~mol}$ hydrazine sulphate are added to $155 \mathrm{~g}$ PPA. The mixture is homogenized under stirring and heated to the reaction temperature of $100-175^{\circ} \mathrm{C}$ and maintained at this temperature for $10-21 \mathrm{~h}$. The reaction medium becomes very viscous with increasing reaction time. The polymer solution is precipitated in hyperfiltrated water and neutralized with sodium hydroxide. The product is repeatedly washed with hyperfiltrated water and dried in a vacuum oven at $60^{\circ} \mathrm{C}$. The polymers were obtained in a quantitative yield.

\section{Preparation of Homogeneous Films}

\section{Poly-1,2,4-triazoles}

Homogeneous poly-1,2,4-triazole films are prepared from a $15 \mathrm{wt} \%$ polymer solution in formic acid. Inhomogenities are removed from this solution by filtration over a $5 \mu \mathrm{m}$ filter. The solution is cast on a glass plate using casting knives with slit heights of 0.3 and $0.5 \mathrm{~mm}$, followed by evaporation of the solvent in a nitrogen atmosphere at room temperature. After evaporation, the glass plate is immersed into a water bath and the poly-1,2,4-triazole film is easily removed from the glass plate. Transparent films are obtained with thicknesses of $20-60 \mu \mathrm{m}$. The films are dried in a vacuum oven for at least 24 h at $150^{\circ} \mathrm{C}$.

\section{Poly-1,2,4-oxadiazoles}

For PIDA-POD and HF-POD the same procedure was followed but in this case trifluoro acetic acid was used as a solvent. For CH-POD, DPE-POD, and TBI-POD, formic acid was used while for the 50/50 copolymer HF/DPE-POD, NMP was used as a solvent, which was evaporated at $150^{\circ} \mathrm{C}$. 


\section{Characterization}

\section{Inherent Viscosity}

The inherent viscosities are determined for $0.5 \mathrm{~g}$ polymer solutions in $100 \mathrm{~mL}$ solvent at $30^{\circ} \mathrm{C}$. For the polyhydrazides, DMSO is used as a solvent, while, for the poly-1,2,4-triazoles and poly-1,3,4-oxadiazoles, formic acid was used. The inherent viscosities of PIDA-POD and HF-POD were determined in concentrated sulphuric acid, while, for the copolymer HF / DPE-POD, NMP was used as a solvent.

\section{Differential Scanning Calorimetry (DSC)}

DSC measurements are performed on a Perkin-Elmer DSC-4 in combination with a System 4 Microprocessor Controller and a Thermal Analysis Data Station (TADS), model 3700 . The polymer samples are placed in aluminium sample pans, and the temperature is increased with a heating rate of $20^{\circ} \mathrm{C} /$ min from $100^{\circ} \mathrm{C}$ to $400^{\circ} \mathrm{C}$ under a nitrogen purge gas stream. The sample is cooled with $320^{\circ} \mathrm{C} / \mathrm{min}$ to $100^{\circ} \mathrm{C}$ and a second run is recorded from $100^{\circ} \mathrm{C}$ to $450^{\circ} \mathrm{C}$ again with a heating rate of $20^{\circ} \mathrm{C} / \mathrm{min}$, as glass transition temperature $\left(T_{g}\right)$, the midpoint of the enthalpy change, is taken, which has been calculated by means of the TADS software.

\section{Thermogravimetrical Analysis (TGA)}

TGA experiments are carried out on a Perkin-Elmer TGS-2 in combination with a System 4 Microprocessor Controller and a Thermal Analysis Data Station (TADS), model 3700. Experiments are carried out under a nitrogen purge gas stream from 50 to $600^{\circ} \mathrm{C}$. The temperature of the polymer samples is increased with a heating rate of $20^{\circ} \mathrm{C} / \mathrm{min}$ and the weight loss has been monitored.

The degradation temperature $\left(T_{\mathrm{deg}}\right)$, defined as the point of intersection of the tangents, in the thermogram was taken (see Fig. 2).

\section{RESULTS AND DISCUSSION}

\section{Poly-1,2,4-triazoles via Polyhydrazides}

The preparation of high molecular weight poly-1,2,4triazoles using polyhydrazide as a precursor polymer was originally reported by Holsten and Lilyquist. ${ }^{6}$ The reaction is carried out in PPA containing an

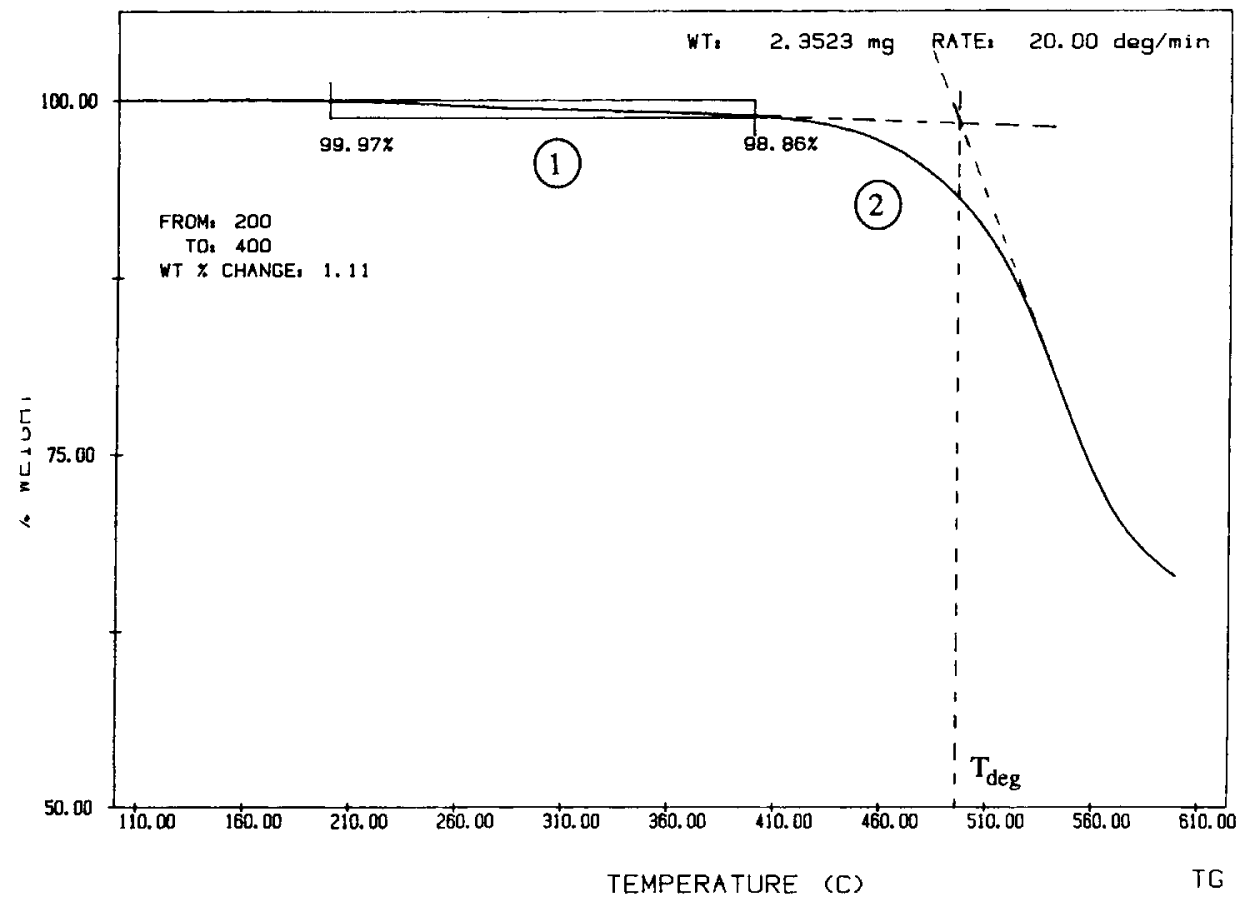

Figure 2. TGA thermogram of $\mathrm{PT}-\mathrm{p} / \mathrm{m}-25 \mathrm{~F}$ recorded with a heating rate of $20^{\circ} \mathrm{C} / \mathrm{min}$ from 100 to $600^{\circ} \mathrm{C}$ under nitrogen. Two phases of weight loss can be observed: (1) loss of water caused by the cyclodehydration of unclosed groups and (2) loss of volatiles caused by the degradation of the polymer. The degradation temperature $\left(T_{\mathrm{deg}}\right)$ is defined as the intersection of the tangents. 
excess of aniline. The ideal concentrations of the reactants were determined to be $10 \mathrm{~g}$ polyhydrazide and $0.823 \mathrm{~mol}$ aniline per $310 \mathrm{~g}$ PPA.

We have incorporated aniline in combination with aniline derivatives and studied the effect on the physical properties of the polymers prepared. The effects on the gas separation properties of these poly-1,2,4-triazoles are reported elsewhere. ${ }^{2}$

Poly-1,2,4-triazoles were prepared from two batches of polyhydrazides having inherent viscosities of 0.86 and $0.82 \mathrm{dL} / \mathrm{g}$.

\section{Elemental Analysis}

Elemental analysis of $\mathrm{C}, \mathrm{H}$, and $\mathrm{N}$ were carried out. In Table $\mathrm{I}$, the $\mathrm{C} / \mathrm{N}$ and $\mathrm{C} / \mathrm{H}$ ratio of the elemental analysis of the polyhydrazides and the poly-1,2,4triazoles are given.

The experimental values found were somewhat lower than the theoretical ones. Nevertheless, the $\mathrm{C} / \mathrm{N}$ and $\mathrm{C} / \mathrm{H}$ ratios are close to their theoretical values indicating residual impurities, most likely to be polyphosphoric acid. ${ }^{13}$ Polyhydrazide and poly1,2,4-triazole are prepared reproducibly, as is indicated by the elemental analysis of $\mathrm{PH}-\mathrm{p} / \mathrm{m}-1, \mathrm{PH}-$ $\mathrm{p} / \mathrm{m}-2, \mathrm{PT}-\mathrm{p} / \mathrm{m}-1, \mathrm{PT}-\mathrm{p} / \mathrm{m}-2$, and $\mathrm{PT}-\mathrm{p} / \mathrm{m}-3$.

The chlorine contents of PT-p $/ \mathrm{m}-25 \mathrm{Cl}$ and PT$\mathrm{p} / \mathrm{m}-50 \mathrm{Cl}$ were determined to be 4.52 and $8.21 \mathrm{wt}$ $\%$, respectively. This indicates that incorporation of chloro aniline is favored over aniline itself, since incorporation based on stoichiometric concentrations in the reaction medium would have yielded theoretical chlorine values of 3.89 and $7.5 \mathrm{wt} \%$, respectively.

Aniline or its derivatives forms a complex with PPA. This complex is in equilibrium with the free amines. Since only free amines can react with polyhydrazide, relatively more free chloro aniline must be available than can be accounted for on the basis of the ratio of aniline/chloro aniline present in the reaction medium. This means that equilibrium between complex and free amine has shifted in the direction of the latter one being chloro aniline. Similar incorporations are assumed to have taken place in the case of PT-p/m-75Cl, PT-p/m-25F, and PT$\mathrm{p} / \mathrm{m}-50 \mathrm{~F}$.

\section{Cyclodehydration}

The results obtained with TGA and DSC techniques are given in Table II. In Figure 2, a TGA run of PT$\mathrm{p} / \mathrm{m}-25 \mathrm{~F}$ is represented, and in Figure 3, two successive DSC curves of this poly-1,2,4-triazole are represented.

Unclosed 1,3,4-oxadiazole and 1,2,4-triazole groups can be present in the final product. During a TGA experiment a distinct weight loss is observed, phase 1 in Figure 2, attributable to the loss of water formed during the cyclodehydration of 1,3,4-oxadi-

Table I. Inherent Viscosities and Elemental Analyses of Polyhydrazides and Poly-1,2,4-triazoles

\begin{tabular}{|c|c|c|c|c|c|c|}
\hline Polymer & $\begin{array}{l}\text { Contents Aniline/ } \\
\text { Aniline Derivative }^{\mathrm{a}}\end{array}$ & $\begin{array}{c}\eta_{\mathrm{inh}}^{\mathrm{b}} \\
(\mathrm{dL} / \mathrm{g})\end{array}$ & $\begin{array}{c}\mathrm{C} / \mathrm{N}^{\mathrm{c}} \\
\text { (Found) }\end{array}$ & $\begin{array}{c}\mathrm{C} / \mathrm{N}^{\mathrm{c}} \\
\text { (Theor.) }\end{array}$ & $\begin{array}{c}\mathrm{C} / \mathrm{H}^{c} \\
\text { (Found) }\end{array}$ & $\begin{array}{c}\mathrm{C} / \mathrm{H}^{\mathrm{c}} \\
\text { (Theor.) }\end{array}$ \\
\hline $\mathrm{PH}-p / m-1$ & & 0.82 & 3.50 & 3.43 & 15.66 & 15.89 \\
\hline PH-p $/ m-2$ & & 0.86 & 3.50 & 3.43 & 14.32 & 15.89 \\
\hline $\mathrm{PT}-p / m-1$ & $100 \%$ aniline & 0.85 & 4.05 & 4.00 & 17.99 & 18.53 \\
\hline $\mathrm{P} T-\mathrm{p} / m-2$ & $100 \%$ aniline & 0.94 & 4.06 & 4.00 & 19.42 & 18.53 \\
\hline $\mathrm{PT}-p / m-3$ & $100 \%$ aniline & 1.01 & 4.13 & 4.00 & 19.57 & 18.53 \\
\hline $\mathrm{PT}-p / m-25 \mathrm{Cl}$ & $25 \% p-\mathrm{Cl}$ & 0.94 & 4.07 & 4.00 & 17.09 & 19.07 \\
\hline $\mathrm{PT}-p / m-50 \mathrm{Cl}$ & $50 \% p-\mathrm{Cl}$ & 0.80 & 4.08 & 4.00 & 16.97 & 19.64 \\
\hline PT-p/m-75Cl & $75 \% p-\mathrm{Cl}$ & 0.80 & 4.08 & 4.00 & 20.70 & 20.24 \\
\hline $\mathrm{PT}-p / m-100 \mathrm{Cl}$ & $100 \% p-\mathrm{Cl}$ & 0.78 & 4.16 & 4.00 & 21.62 & 20.84 \\
\hline $\mathrm{PT} \cdot p / m-25 \mathrm{~F}$ & $25 \% p-\mathrm{F}$ & 0.99 & 4.07 & 4.00 & 19.27 & 19.08 \\
\hline $\mathrm{PT}-p / m-50 \mathrm{~F}$ & $50 \% p-\mathrm{F}$ & 1.10 & 4.05 & 4.00 & 19.11 & 19.65 \\
\hline PT-p/m-100F & $100 \% p-\mathbf{F}$ & 0.95 & 4.07 & 4.00 & 19.95 & 20.85 \\
\hline $\mathrm{PT}-p / m-p-\mathrm{Me}$ & $100 \% p-\mathrm{Me}$ & 1.12 & 4.36 & 4.28 & 17.08 & 16.26 \\
\hline $\mathrm{PT}-p / m-o-\mathrm{Me}$ & $100 \% o-\mathrm{Me}$ & 0.98 & 4.21 & 4.29 & 17.44 & 16.26 \\
\hline
\end{tabular}

${ }^{a}$ Proportions aniline/aniline derivative added to the reaction medium, for example PT- $p / m-75 \mathrm{Cl}$ means that a mixture consisting of $25 \%$ aniline and $75 \% p$-chloro aniline is applied, $p$-F stands for $p$-fluoro aniline, $p$-Me is $p$-toluidine, and $o$-Me is $o$-toluidine.

${ }^{\mathrm{b}}$ Inherent viscosities were determined from polymer solutions $(0.5 \mathrm{~g} / \mathrm{dL})$ at $30^{\circ} \mathrm{C}$. DMSO was used as solvent in the case of polyhydrazide and formic acid in the case of the poly-1,2,4-triazoles.

${ }^{\mathrm{c}}$ Determined using elemental analysis (wt \%/wt \%). 
Table II. Film Appearance and Thermal Properties of the Poly-1,2,4-triazoles

\begin{tabular}{|c|c|c|c|c|c|c|c|}
\hline \multirow[b]{2}{*}{ Polytriazole } & \multirow[b]{2}{*}{$\begin{array}{c}\text { Film } \\
\text { Appearance }\end{array}$} & \multicolumn{2}{|c|}{$T_{g}^{\mathrm{b}}$} & \multirow[b]{2}{*}{$\begin{array}{l}T_{c c}{ }^{c} \\
\left({ }^{\circ} \mathrm{C}\right)\end{array}$} & \multirow[b]{2}{*}{$\begin{array}{l}T_{m}{ }^{d} \\
\left({ }^{\circ} \mathrm{C}\right)\end{array}$} & \multirow[b]{2}{*}{$\begin{array}{l}T_{\text {deg }} \\
\left({ }^{\circ} \mathrm{C}\right)\end{array}$} & \multirow[b]{2}{*}{$\begin{array}{c}\Delta w \\
\left(200-400^{\circ} \mathrm{C}\right) \\
(\text { wt } \%)\end{array}$} \\
\hline & & $\begin{array}{c}1^{\text {st }} \text { Run } \\
\left({ }^{\circ} \mathrm{C}\right)\end{array}$ & $\begin{array}{c}2^{\text {nd }} \text { Run } \\
\left({ }^{\circ} \mathrm{C}\right)\end{array}$ & & & & \\
\hline $\mathrm{PT}-p / m-1$ & Light yellow & 264 & $-\mathrm{e}$ & 319 & 425 & 527 & 2.07 \\
\hline PT-p $/ m-2$ & Light yellow & 267 & 282 & 324 & 427 & 524 & 1.65 \\
\hline $\mathrm{PT}-p / m-3$ & Light yellow & 261 & $-^{\mathbf{e}}$ & 315 & 425 & 521 & 1.82 \\
\hline $\mathrm{PT}-p / m-25 \mathrm{Cl}$ & Yellow & 271 & 288 & $-\mathbf{e}$ & $-e$ & 504 & 1.52 \\
\hline PT- $p / m-50 \mathrm{Cl}$ & Deep yellow & 269 & 276 & $-{ }^{e}$ & $-e$ & 489 & 2.30 \\
\hline $\mathrm{PT}-p / m-75 \mathrm{Cl}$ & Blue & 271 & 278 & $-{ }^{e}$ & $-{ }^{e}$ & 484 & 1.45 \\
\hline $\mathrm{PT}-p / m-100 \mathrm{Cl}$ & Deep blue & 274 & 279 & 373 & 423 & 486 & 1.77 \\
\hline PT- $p / m-25 \mathrm{~F}$ & Light yellow & 257 & 266 & 344 & 407 & 507 & 1.11 \\
\hline $\mathrm{PT}-p / m-50 \mathrm{~F}$ & Light yellow & 269 & 273 & $-e^{e}$ & $-\mathrm{e}$ & 479 & 1.13 \\
\hline PT- $p / m-100 \mathrm{~F}$ & Light yellow & 276 & 283 & $-e$ & 409 & 453 & 1.10 \\
\hline PT- $-p / m-p-\mathrm{Me}$ & Light yellow & 257 & 254 & $-\mathbf{e}$ & $-{ }_{-}^{e}$ & 506 & 1.78 \\
\hline $\mathbf{P T}-p / m-o-\mathbf{M e}$ & Light yellow & 268 & 272 & $-e$ & $-e$ & 493 & 2.88 \\
\hline
\end{tabular}

a DS curves were recorded twice using the same sample. The first run from 100 to $400^{\circ} \mathrm{C}$ and the second run from 100 to $450^{\circ} \mathrm{C}$. In all cases a heating rate of $20^{\circ} \mathrm{C} / \mathrm{min}$ was used. After the first run the sample was cooled down with $320^{\circ} \mathrm{C} / \mathrm{min}$.

${ }^{b}$ All films were transparent except $\mathrm{PT}-p / m-100 \mathrm{~F}$, which was opaque.

c The peak temperature of cold crystallization is given.

d The onset of the melting peak is given.

e No $T_{g}$, cold crystallization, or melting peak detectable using DSC techniques.

azole and 1,2,4-triazole groups. The reaction scheme of this ring closure is represented in Figure 4.

Weight loss occurring between $200^{\circ} \mathrm{C}$ and $400^{\circ} \mathrm{C}$ has been attributed to the ring closure reaction. In all cases, weight losses due to ring closure reactions are observed (see Table II), indicating that the reaction between aniline, aniline derivatives, and polyhydrazide at $175^{\circ} \mathrm{C}$ does not result in a fully converted product. Performing the reaction at higher

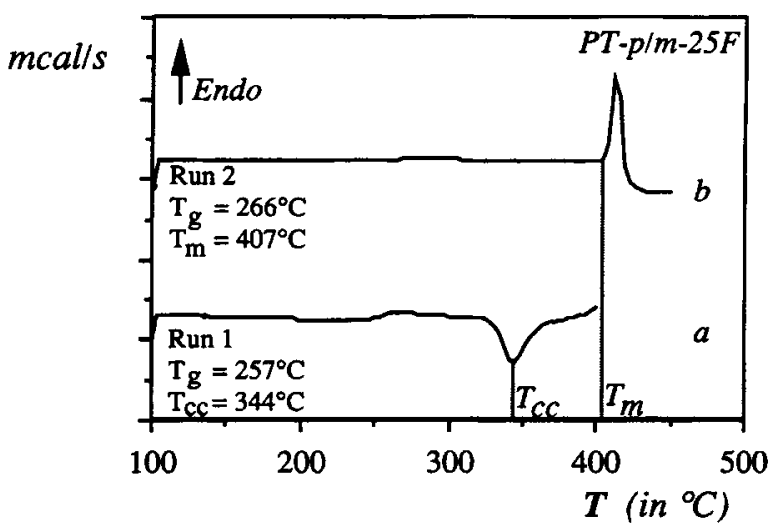

Figure 3. Two successive DSC curves of PT-p/m-25F recorded with $20^{\circ} \mathrm{C} / \mathrm{min}$ under nitrogen. After the first run from 100 to $400^{\circ} \mathrm{C}$ (a) the sample is cooled with $320^{\circ} \mathrm{C} / \mathrm{min}$ and the second run from 100 to $450^{\circ} \mathrm{C}$ is recorded (b). $T_{c c}$ is the peak temperature of cold crystallization and $T_{m}$ is the on-set temperature of melting. temperatures results, however, in products with lower inherent viscosities. ${ }^{6}$ In a thermogram of a fully converted poly-1,2,4-triazole this weight loss is, of course, absent. PT-p/m-o-Me shows the highest weight loss. In this case, the reaction between $o-$ toluidine and polyhydrazide is most probably sterically hindered. Unclosed groups are also detectable by IR-spectrometry, since a carbonyl stretching absorption peak is observed in the IR-spectra at $1650 \mathrm{~cm}^{-1}$.

Unclosed rings are possible weak spots for thermal or chemical degradation. A heat treatment will force unclosed rings into their more thermally stable and chemically resistant closed ring forms.

Phase 2 in Figure 2 represents the loss of volatiles caused by the ultimate degradation of the polymer. Incorporation of aniline derivatives yields polymers with a slightly decreased thermal stability as compared to unmodified poly $[p-, m$-phenylene- (4-

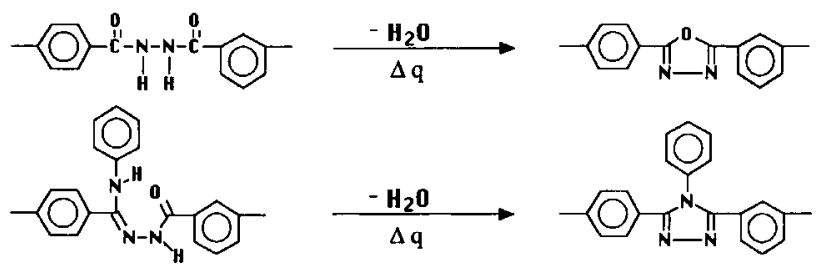

Figure 4. Cyclodehydration of unclosed 1,3,4-oxadiazole and 1,2,4-triazole groups. 
phenyl)-1,2,4-triazoles] ( see Table II). From all polymers, except from PT-p/m-o-Me and PT-p/m$100 \mathrm{~F}$, flexible films were obtained by solvent casting techniques and these were investigated for their gas separation properties. ${ }^{2}$ These films were also used for DSC experiments.

Each sample was subjected to two subsequent DSC heating runs, the first from 100 to $400^{\circ} \mathrm{C}$ after which the sample was cooled down within $320^{\circ} \mathrm{C} /$ min, followed by a second heating run from 100 to $450^{\circ} \mathrm{C}$ ( see Fig. 3 ). In both experiments, a heating rate of $20^{\circ} \mathrm{C} / \mathrm{min}$ was applied. The glass transition temperatures recorded during both runs are given in Table II.

The glass transition temperature is raised with the incorporation of fluoro and chloro aniline, obviously due to increased polar interactions. Incorporation of $o$-toluidine results in a decreased glass transition temperature compared to the unmodified poly-1,2,4-triazoles, PT-p/m-1, PT-p/m-2, and PT$\mathrm{p} / \mathrm{m}-3$. Differences in glass transition temperatures are, however, small.

Except for PT-p/m-p-Me, the glass transition temperature of the second run is higher than the glass transition temperature of the "as-cast" film. This rise in glass transition temperature is obviously due to quenching effects during cooling, ${ }^{11}$ and due to cyclodehydration of unclosed groups during the first DSC experiment in which a more flexible structure is converted into a rigid one. The effect of both quenching and ring closure on the glass transition temperature is nicely shown by the following experiment where a sample of PT-p $/ \mathrm{m}-50 \mathrm{Cl}$ is subjected to a number of successive DSC heating runs, ( see Table III). PT-p/m-50Cl was chosen for this experiment, since it has a relatively large number of unclosed rings, as indicated by its weight loss during the TGA experiments.

During the first run up to $300^{\circ} \mathrm{C}$, a glass transition temperature of $268.5^{\circ} \mathrm{C}$ is measured. After cooling down with $320^{\circ} \mathrm{C} / \mathrm{min}$, the second run is recorded and the $T_{g}$ has slightly increased, obviously due to quenching of the sample during cooling. But further changes in runs 2,3 , and 4 are marginal. Due to the low end temperatures, no significant cyclodehydration reactions of the unclosed rings take place. Increasing the end temperature to $400^{\circ} \mathrm{C}$ results in a rise in $T_{g}$. During these runs the polymer undergoes cyclodehydration resulting in a more rigid polymer. This implies that the greater part of the ring closure reactions takes place when the polymer is in the rubbery phase. During runs 6 and 7 the polymer is likely to undergo also some degradation.

\section{Cold Crystallization}

Cold crystallization is detected for a number of poly1,2,4-triazoles during the first heating run of a DSC experiment. Cold crystallization of alternating poly [ $p$-, $m$-phenylene- (4-phenyl) -1,2,4-triazole ] has extensively been studied by Gebben et al., who noticed that cold crystallization is observed only for poly-1,2,4-triazole films and not for the as-prepared reaction powder. ${ }^{12}$ The process of cold crystallization also depends highly on the macromolecular structure of the polymers used. For example, cold crystallization is observed only for a poly $[p-, m$-phenylene(4-phenyl)-1,2,4-triazole] where $p$ - and $m$-phenylene groups are present in an alternating fashion, and not if these groups are incorporated randomly. ${ }^{13}$ A highly regular macromolecule is obviously a precondition for cold crystallization. A similar phenomenon is observed for the modified poly-1,2,4triazoles as represented in Table II.

During the first DSC heating cycle, a number of poly-1,2,4-triazoles undergo cold crystallization in the rubbery phase. After heating to $300^{\circ} \mathrm{C}$ the polymer is quenched with $320^{\circ} \mathrm{C} / \mathrm{min}$ to $100^{\circ} \mathrm{C}$ and subjected to a second heating cycle to $450^{\circ} \mathrm{C}$. During cold crystallization the amorphous fraction in a polymer decreases, which results in a decreased heat capacity jump at the glass transition temperature. In the case of PT-p/m-1 and PT-p/m-2, this occurs to such an extent that the glass transition temper-

Table III. Influence of Subsequent Heating Runs on the Glass Transition Temperature of PT- $p / m-50 \mathrm{Cl}$

\begin{tabular}{cccccc}
\hline Heating Run & Limits & $\begin{array}{c}\text { Glass Transition } \\
\text { Temperature } \\
\left({ }^{\circ} \mathrm{C}\right)\end{array}$ & Heating Run & Limits & $\begin{array}{c}\text { Glass Transition } \\
\text { Temperature } \\
\left({ }^{\circ} \mathrm{C}\right)\end{array}$ \\
\hline 1 & $100-300$ & 268.5 & 5 & $100-420$ & 278.6 \\
2 & $100-300$ & 271.0 & 6 & $100-455$ & 282.7 \\
3 & $100-300$ & 271.1 & 7 & $100-455$ & 289.3 \\
4 & $100-420$ & 270.6 & & & \\
\hline
\end{tabular}


ature is not detectable in the DSC curve of the second run. The crystalline fraction in poly-1,2,4-triazoles formed during cold crystallization melts in the second run as indicated by a clear melting peak [ see Fig. 3 (B) ]. The onset of this melting peak is given, since the peak temperature could not be indicated clearly in a number of cases due to simultaneously occurring degradation.

As said, the unmodified poly-1,2,4-triazoles undergo cold crystallization in the rubbery phase. Incorporation of $p$-chloro aniline in combination with aniline results in a less regular macromolecular structure and the possibility of undergoing cold crystallization vanishes. In the case of PT-p/m$100 p-\mathrm{Cl}$ the polymer becomes "regular" again and crystallization is observed. PT-p/m-25F undergoes cold crystallization indicating that incorporation of $25 \% p$-fluoro aniline does not obviously result in a dramatic change in the macromolecular structure, due to the small amount and the relative small size of the fluorine atom. No cold crystallization is observed for PT-p/m-100F. Since the appearance of the "as-cast" polymer film is opaque, and since a melting peak is observed with an onset of $453^{\circ} \mathrm{C}$, this polymer is believed to be already crystalline.

One also might expect cold crystallization for the regular $\mathrm{PT}-\mathrm{p} / \mathrm{m}-\mathrm{p}-\mathrm{Me}$, but no cold crystallization or melting peak is observed in the DSC curve. The reason for this behavior is not understood.

\section{Other Pendant Groups}

Since solubility is often enhanced when larger groups are incorporated onto the polymer backbone as pendant groups, we have investigated the possibility of incorporating biphenylic structures, like 1-naphthylamine, 4-phenoxy aniline, and 4-aminobenzotrifluoride, but in this case polymers with insufficient film-forming properties were obtained. During the reaction between amine and polyhydrazide, polymer degradation occurs due to the strong acidic environment of polyphosphoric acid. This degradation is kept to minimum if the reaction medium consists of 0.832 mol amine per $310 \mathrm{~g} \mathrm{PPA} .^{6}$ It, however, was not possible to dissolve these amounts of these high molecular weight amines in PPA. Another problem was the sublimation of these amines from the reaction mixture during the reaction, resulting in a further deviation from the ideal reaction conditions. Similar problems have been reported by Krongauz et al., ${ }^{14}$ who investigated the reaction between 1 naphtylamine and poly ( $p$-phenylene-1,3,4-oxadiazole) also in PPA.
Incorporation of cyclohexylamine was also examined. During this reaction, only minor quantities of polyhydrazide are converted into the corresponding poly-1,2,4-triazole. In the case of a normal reaction behavior, the precursor dissolves completely in the course of the reaction and is converted into the corresponding poly-1,2,4-triazole. Due to the strong complex formation between polyphosphoric acid and cyclohexylamine ${ }^{14}$ the concentration of free amines is apparently too low to result in a successful reaction.

\section{Poly-1,3,4-oxadiazoles}

Poly ( $p$-, $m$-phenylene ) -1,3,4-oxadiazoles are comparable with poly ( $p$-phenylene) with respect to their thermal stability and chemical resistance, which is ascribed to the fact that the 1,3,4-oxadiazole ring is spectrally and electronically equivalent to the $p$ phenylene ring. ${ }^{15}$ High thermal stability and chemical resistance is favored in most end-use applications. During processing, these characteristics can, however, be a major problem.

Poly ( $p$-, $m$-phenylene) -1,3,4-oxadiazole can be obtained via a cyclodehydration reaction of the corresponding tractable polyhydrazide. However, solid state cyclodehydration of alternating poly $(p-, m$ phenylene hydrazide ) yielded brittle membranes. ${ }^{13}$

Poly ( $p$-, $m$-phenylene)-1,3,4-oxadiazoles prepared from isophthalic acid and terephthalic acid with hydrazine sulphate as monomers yielded polymers with higher inherent viscosities from which flexible membranes were prepared. Since this reaction gave poly-1,3,4-oxadiazoles with superior properties, it was also used to prepare poly-1,3,4oxadiazoles with various other functional groups. The physical properties of these polymers were studied as a function of these functional groups. The poly-1,3,4-oxadiazoles were prepared from various dicarboxylic acids ( see Fig. 1). These poly-1,3,4-oxadiazoles were, in turn, used as precursor polymers for the preparation of poly-1,2,4-triazole on which we hope to report in the future.

Fuming sulphuric acid is mostly favored over PPA as a reaction medium for the preparation of aromatic poly-1,3,4-oxadiazoles. ${ }^{16}$ But in the case of 1,1,3-trimethyl-3- ( $p$-carboxy-phenyl )-5-carboxyindan (PIDA), no polymer was obtained, possibly due to degradation of the monomer that contains aliphatic groups obviously susceptible to degradation in the strong acidic environment. Performing the reaction in PPA, a much milder dehydrating agent, resulted readily in a very viscous reaction medium and gave 
Table IV. Elemental Analysis of Poly-1,3,4-oxadiazoles

\begin{tabular}{llccccc}
\hline \multicolumn{1}{c}{ Polymer } & $\begin{array}{c}\text { Dicarboxylic } \\
\text { Acid }\end{array}$ & $\begin{array}{c}\eta_{\text {inh }}{ }^{\mathrm{a}} \\
(\mathrm{dL} / \mathrm{g})\end{array}$ & $\begin{array}{c}\mathrm{C} / \mathrm{N} \\
\text { (Found) }\end{array}$ & $\begin{array}{c}\mathrm{C} / \mathrm{N} \\
\text { (Theor.) }\end{array}$ & $\begin{array}{c}\mathrm{C} / \mathrm{H} \\
\text { (Found) }\end{array}$ & $\begin{array}{c}\mathrm{C} / \mathrm{H} \\
\text { (Theor.) }\end{array}$ \\
\hline CH-POD & $c$-CH & 1.65 & 3.46 & 3.43 & 9.74 & 9.54 \\
$t$-CH-POD & $t$-CH & 2.81 & 3.61 & 3.43 & 8.94 & 9.54 \\
$c+t$-POD & $c+t$-CH & 1.56 & 3.42 & 3.43 & 9.54 \\
PIDA-POD & PIDA & $0.19^{\mathbf{b}}$ & 8.63 & 8.58 & 12.75 & 12.55 \\
TBI-POD & TBI & 0.38 & 5.64 & 5.15 & 11.65 & 11.92 \\
HF-POD & HF & $0.40^{\mathbf{b}}$ & 7.50 & 7.29 & 25.38 & 25.30 \\
DPE-POD & DPE & 0.37 & 6.05 & 6.00 & 15.38 & 20.87 \\
HF/DPE-POD & HF \& DPE & $2.44^{\mathbf{c}}$ & 6.26 & 6.64 & 16.88 & 23.08 \\
\hline
\end{tabular}

anherent viscosity is determined in formic acid.

${ }^{\mathrm{b}}$ Inherent viscosity is determined in concentrated sulphuric acid.

' Inherent viscosity is determined in NMP.

${ }^{\mathrm{d}}$ Random copolymer of $\mathrm{HF}$ and DPE used in $40 \%$ and $60 \%$ molar amounts, theoretical values for the polymer elemental analysis were calculated assuming stoichiometric incorporation of both monomers.

a polymer with an inherent viscosity of $0.19 \mathrm{dL} / \mathrm{g}$ from which flexible films were prepared. To prevent monomer degradation, all syntheses represented in Figure 1 (B) were performed in PPA instead of fuming sulphuric acid.

Successful preparation of poly-1,3,4-oxadiazoles was confirmed by elemental analysis ( see Table IV). Also in this case, the experimental $\mathrm{C}, \mathrm{N}$, and $\mathrm{H}$ values found were somewhat lower than the theoretical ones. Nevertheless, the $\mathrm{C} / \mathrm{N}$ and $\mathrm{C} / \mathrm{H}$ ratios are close to their theoretical values indicating residual impurities in the final product, most likely to be polyphosphoric acid. ${ }^{13}$ Flexible films were obtained from all poly-1,3,4-oxadiazoles by solvent casting techniques.

In Table $\mathrm{V}$, the glass transition temperatures and the degradation temperatures are given. Again samples are subjected to two successive heating runs, the first from 100 to $400^{\circ} \mathrm{C}$, after which the sample is cooled down with $320^{\circ} \mathrm{C} / \mathrm{min}$ to $100^{\circ} \mathrm{C}$, followed by a second run with an end temperature of $450^{\circ} \mathrm{C}$. Both scans were recorded with a heating rate of $20^{\circ} \mathrm{C} / \mathrm{min}$. These poly-1,3,4-oxadiazoles all have high glass transition temperatures. The first run yields the glass transition temperature of the asprepared film. The glass transition temperature recorded during the second run is higher than the first. In this case, quenching effects and cyclodehydration of unclosed hydrazide groups into the more rigid 1,3,4-oxadiazole groups probably occur (see Fig. 4). In the second run, the glass transition temperature of a number of poly-1,3,4-oxadiazoles were not detectable with DSC techniques.

The thermal stability of these poly-1,3,4-oxadiazoles is slightly lower than those of the poly-1,2,4- triazoles. Discriminating between weight loss due to cyclodehydration and degradation is in this case not possible to full satisfaction, since these two phases of weight loss shade off into one another.

\section{CH-POD}

Preparation of alicyclic poly-1,3,4-oxadiazoles was originally reported by Iwakura et al. ${ }^{9}$ Poly (1,4-cyclohexane ) -1,3,4-oxadiazole was reported to be soluble in formic acid while good thermal stability was maintained. Incorporation of the flexible cyclohexane unit results in a decrease of the glass transition temperature compared to its aromatic counterpart, poly ( $p$-phenylene ) -1,3,4-oxadiazole. The degradation temperature is approximately $100^{\circ} \mathrm{C}$ lower than

Table V. Film Appearance and Thermal Properties of Poly-1,3,4-oxadiazoles

\begin{tabular}{|c|c|c|c|c|}
\hline \multirow[b]{2}{*}{ Polymer } & \multirow[b]{2}{*}{$\begin{array}{c}\text { Film } \\
\text { Appearance }\end{array}$} & \multicolumn{2}{|c|}{$T_{g}$} & \multirow[b]{2}{*}{$\begin{array}{l}T_{\mathrm{deg}} \\
\left({ }^{\circ} \mathrm{C}\right)\end{array}$} \\
\hline & & $\begin{array}{c}1^{\text {st }} \text { Run } \\
\left({ }^{\circ} \mathrm{C}\right)\end{array}$ & $\begin{array}{c}2^{\text {nd }} \text { Run } \\
\left({ }^{\circ} \mathrm{C}\right)\end{array}$ & \\
\hline$c$-CH-POD & Light yellow & 179 & $-{ }^{a}$ & 441 \\
\hline$t$-CH-POD & Colorless & 179 & $--^{a}$ & 449 \\
\hline$c+t-\mathrm{POD}$ & Colorless & 180 & $-{ }^{a}$ & 449 \\
\hline PIDA-POD & Light yellow & 300 & 330 & 488 \\
\hline TBI-POD & Colorless & 315 & $--^{a}$ & 485 \\
\hline HF-POD & Colorless & 287 & 295 & 479 \\
\hline DPE-POD & Colorless & 333 & 361 & 496 \\
\hline HF/DPE-POD & Light yellow & 339 & $-{ }^{a}$ & 491 \\
\hline
\end{tabular}

\footnotetext{
${ }^{a}$ No glass transition temperature detectable with DSC tech-
} niques. 
found for poly ( $p$-phenylene ) -1,3,4-oxadiazole, being $542^{\circ} \mathrm{C}^{13}$ Our results on the thermal stability of $\mathrm{CH}$ POD are in agreement with the observations of Iwakura et al. ${ }^{9}$

Cyclohexane-1,4-dicarboxylic acid was supplied as a mixture of cis and trans isomers. Both this mixture as well as the pure cis-and trans-isomers were used as monomers, resulting in three batches of poly (1,4-cyclohexane) -1,3,4-oxadiazole. Flexible films were obtained from all three polymers. No differences were detected between the three batches using elemental analysis, IR-spectroscopy, and DSC techniques.

Iwakura synthesized two batches of poly (1,4-cyclohexane )-1,3,4-oxadiazole using the cis- and transisomers of 1,4-cyclohexane dicarboxylic acid and concluded that the two polymers had the same geometrical structure on the basis of their identical infrared spectrum, solubility, thermal stability, and crystallinity. He assumed that cis-1,4-cyclohexane was converted into the more stable trans isomer in the acidic environment of PPA. ${ }^{9}$ The identical glass transition temperatures of our three batches of poly (1,4-cyclohexane)-1,3,4-oxadiazole endorses this assumption.

\section{PIDA-POD}

The 1,1,3-trimethyl-3-phenylindane unit is used as a solubility increasing unit for a number of polymers. For example, Ciba-Geigy's soluble polyimide "XU218 " also contains this bulky and asymmetrical moiety. We have investigated whether incorporation of this unit had the same effect on solubility behavior of the poly-1,3,4-oxadiazoles. Compared to the fully aromatic poly ( $p, m$-phenylene)-1,3,4-oxadiazoles, the solubility is increased. PIDA-POD is, beside its solubility in concentrated sulphuric acid, also soluble in trifluoro acetic acid.

Using DSC techniques, a glass transition temperature of $330^{\circ} \mathrm{C}$ (second run, see Table V) was determined for PIDA-POD, which is comparable with the high $T_{g}$ reported by Wilson ${ }^{17}$ of $341^{\circ} \mathrm{C}$ (also second run) for a polyamide derived from 1,1,3-trimethyl-3-( $p$-aminophenyl $)-5$-indanamine and 1,1,3-trimethyl-3-phenylindan-4',5-dicarboxylic acid.

\section{TBI-POD}

The preparation of TBI-POD using oleum as a reaction mixture has been reported in a patent by Stephens. ${ }^{18}$ The preparation of flexible films from TBI$\mathrm{POD} / m$-cresol solutions is claimed in this patent.
We have initially performed this synthesis in PPA. After solvent casting from formic acid, a polymer with insufficient film-forming properties was obtained, possibly due to the low inherent viscosity. Using a mixture of sulphuric acid and chlorosulphonic acid as a reaction medium resulted in a polymer with a higher inherent viscosity, $0.7 \mathrm{dL} / \mathrm{g}$. Flexible films were obtained from TBI-POD $/ m$ cresol solutions after evaporation of the solvent.

\section{HF-POD}

Incorporation of the polar and bulky $4,4^{\prime}-\left(2,2^{\prime}\right.$-diphenyl ) hexafluoropropane moiety into the polymer backbone of polyimides has shown to be an effective way of substantially increasing the solubility of these polymers. ${ }^{19}$ The HF unit has also been incorporated into the polymer backbone of a poly-1,3,4-oxadiazole by Russian workers. ${ }^{20}$ This poly-1,3,4-oxadiazole was prepared by cyclodehydration of a polyhydrazide. The polyhydrazide was, in turn, prepared by a lowtemperature polycondensation of the dihydrazide and dichloride of 4,4'-(2,2'-diphenyl) hexafluoro propane dicarboxylic acid. We have prepared HFPOD directly by the less laborious one-step synthesis using 4,4'-(2,2'-diphenyl) hexafluoro propane dicarboxylic acid and hydrazine as monomers. Flexible films were cast from a HF-POD/trifluoro acetic acid solution.

The Russian workers reported a glass transition temperature of approximately $333^{\circ} \mathrm{C}$ with DTA for their poly-1,3,4-oxadiazole, which is somewhat higher than the value we have found using DSC ( see Table IV). This may be due to the difference in preparation history of the polymer.

Solubility tests showed that HF-POD is soluble in THF, chloroform, NMP, $o$-dichloro benzene, and trifluoro acetic acid. The increased solubility of HFPOD compared to the other poly-1,3,4-oxadiazoles is believed to be a result of the more loosely packed polymer matrix, as was found by a Russian worker for trifluoromethyl containing polyamides ${ }^{20}$ allowing enhanced solvent diffusion into the polymer matrix followed by its subsequent dissolution.

\section{DPE-POD}

Incorporation of the diphenyl ether unit results in a less rigid poly-1,3,4-oxadiazole as compared with the corresponding poly ( $p$-phenylene) -1,3,4-oxadiazole. Elemental analysis indicates a surplus of hydrogen atoms. This deviation from the theoretical value may be due to a combination of the polymers hygroscopic nature and its relative small number of hydrogen atoms in comparison to carbon, resulting in a large relative error. 
A glass transition temperature of $333^{\circ} \mathrm{C}$ was obtained for DPE-POD using DSC techniques; in the second run of the same sample a much broader glass transition area with a midpoint of approximately $361^{\circ} \mathrm{C}$ was found. These observations are in agreement with those of Korshak et al. ${ }^{21}$ DPE-POD exhibits a good thermal stability as indicated by its high degradation temperature. During a TGA experiment, unclosed 1,3,4-oxadiazole groups undergo cyclodehydration.

Flexible films were cast from a polymer solution in formic acid. Beside its solubility in formic acid, DPE-POD is soluble in DMSO and NMP.

\section{HF/DPE-POD}

Since DPE-POD and HF-POD are each other's extremes in permeability and selectivity, ${ }^{2}$ the random copolymer HF /DPE-POD was also prepared using $40 \% / 60 \%(\mathrm{~mol} / \mathrm{mol})$ mixture of both monomers in combination with hydrazine sulphate. Flexible films were cast from a polymer solution in NMP. The glass transition temperature and degradation temperature are in agreement with those of the homopolymers.

\section{CONCLUSIONS}

The glass transition temperature, degradation temperature, and cold crystallization behavior of aromatic poly-1,2,4-triazoles have been studied, where various groups have been introduced onto the pendant phenyl group. Cold crystallization was observed only if macromolecular regularity was maintained to a large extent.

The solubility of poly-1,3,4-oxadiazoles was highly improved with the incorporation of 5-t-butylisophthalic, 1,1,3-trimethyl-3-phenylindane, 4,4'(2,2'-diphenyl) hexafluoro propane, and diphenyl ether groups into the polymer main chain, whereas high glass transition temperatures and degradation temperatures typical for aromatic poly-1,3,4-oxadiazoles were maintained.

The authors wish to thank Dr. E. E. Paschke (Amoco Chemical Company, U.S.A.), Dr. G. Hoentjen and Dr. W. J. Mijs (Akzo Research Laboratories Arnhem, The Netherlands), and Dr. W. Diesing (Hoechst, Germany) for their kind supply of dicarboxylic acids. The authors are indebted to L. A. de Graaf for carrying out a large number of syntheses. Akzo International Research is ac- knowledged for their financial support, and CPNQ Brazil is acknowledged for the support of the research project of M. S.

\section{REFERENCES AND NOTES}

1. B. Gebben, M. H. V. Mulder, and C. A. Smolders, J. Membrane Sci., 46, 29-41 (1989).

2. E. R. Hensema, J. P. Boom, M. E. R. Sena, M. H. V. Mulder, and C. A. Smolders, Polymer, to appear.

3. Y. Iwakura, K. Uno, and S. Hara, Makromol. Chem., 108, 160-169 (1967).

4. A. P. Bogdanov et al., GB 2093.460 (1982).

5. R. J. Cotter and M. Matzner, in A. T. Blomquist and H. Wasserman, (Eds.), Organic Chemistry, Vol. 13B, 1, Academic Press, New York, 1972.

6. J. R. Holsten and M. R. Lilyquist, J. Polym. Sci., A, 3, 3905-3917 (1965)

7. A. H. Frazer and F. T. Wallenberger, J. Polym. Sci., A2, 1137-1145 (1964).

8. Y. Iwakura, K. Uno, and S. Hara, J. Polym. Sci., A, 3, 45-54 (1965).

9. Y. Iwakura, K. Uno, and S. Hara, Makromol. Chem., 95, 248-260 (1966).

10. A. H. Frazer and F. T. Wallenberger, J. Polym. Sci., A2, 1147-1156 (1964).

11. M. J. Richardson and N. G. Savill, Polymer, 16, 753757 ( 1975 ).

12. B. Gebben, M. H. V. Mulder, and C. A. Smolders, $J$. Polym. Sci., Polym. Chem. Ed., 27, 3481-3499 (1989).

13. E. R. Hensema, J. P. Boom, M. H. V. Mulder, and C. A. Smolders, J. Polym. Sci., Polymer Chemistry Ed., to appear.

14. E. S. Krongauz, V. V. Korshak, and A. V. D'Yachenko, Vysokomol. Soedin. Ser. B, 10, 108-111 (1968).

15. J. Sauer, R. Huisgen, and H. J. Sturm, Tetrahedron, 11, $214(1960)$.

16. Y. Iwakura, K. Uno, and S. Hara, Makromol. Chem., 94, 103-113 (1966).

17. J. C. Wilson, J. Polym. Sci., Polymer Chemistry Ed., 13, 749-754 (1975).

18. J. R. Stephens, U.S. Patent 4,487,921, 1984.

19. F. W. Harris and L. H. Lanier, in F. W. Harris and R. B. Seymour (Eds.), Structure-Solubility Relation ships in Polymers, London, Academic Press, 1977.

20. B. R. Livshits, S. V. Vinogradova, I. L. Knunyunts, G. L. Berestneva, and T. K. L. Dymoshits, Vysokomol. Soyed., A15, 961-968 (1973).

21. V. V. Korshak, A. V. D'Yachenko, Ye. S. Krongauz, and G. L. Berestneva, Vysokomol. Soyed. Ser. A, 11, $7-10(1969)$.

Received October 29, 1991

Accepted August 3, 1993 